\title{
Intestinal barrier tightening by a cell-penetrating antibody to Bin1, a candidate target for immunotherapy of ulcerative colitis.
}

\author{
Sunil Thomas \\ Lankenau Institute for Medical Research \\ Kevther Hoxha \\ Lankenau Institute for Medical Research \\ Walker Alexander \\ Lankenau Institute for Medical Research \\ John Gilligan

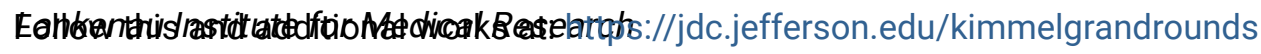

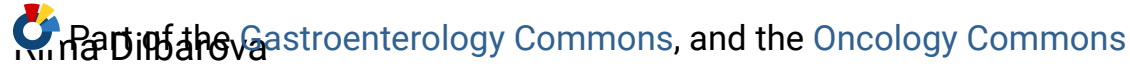

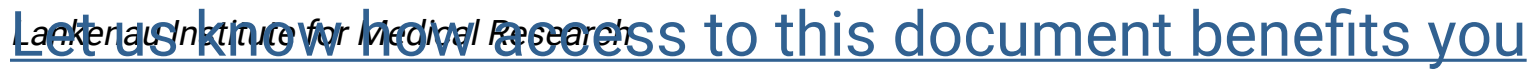

\section{Beconmergerd/Citantional authors}

Thomas, Sunil; Hoxha, Kevther; Alexander, Walker; Gilligan, John; Dilbarova, Rima; Whittaker, Kelly; Kossenkov, Andrew; Prendergast, George C.; and Mullin, James M., "Intestinal barrier tightening by a cell-penetrating antibody to Bin1, a candidate target for immunotherapy of ulcerative colitis." (2018). Kimmel Cancer Center Papers, Presentations, and Grand Rounds. Paper 64.

https://jdc.jefferson.edu/kimmelgrandrounds/64

This Article is brought to you for free and open access by the Jefferson Digital Commons. The Jefferson Digital Commons is a service of Thomas Jefferson University's Center for Teaching and Learning (CTL). The Commons is a showcase for Jefferson books and journals, peer-reviewed scholarly publications, unique historical collections from the University archives, and teaching tools. The Jefferson Digital Commons allows researchers and interested readers anywhere in the world to learn about and keep up to date with Jefferson scholarship. This article has been accepted for inclusion in Kimmel Cancer Center Papers, Presentations, and Grand Rounds by an authorized administrator of the Jefferson Digital Commons. For more information, please contact: JeffersonDigitalCommons@jefferson.edu. 


\section{Authors}

Sunil Thomas, Kevther Hoxha, Walker Alexander, John Gilligan, Rima Dilbarova, Kelly Whittaker, Andrew Kossenkov, George C. Prendergast, and James M. Mullin 


\title{
Intestinal barrier tightening by a cell-penetrating antibody to Bin1, a candidate target for immunotherapy of ulcerative colitis
}

\author{
Sunil Thomas $^{1}$ (D) Kevther Hoxha $^{1}$ | Walker Alexander ${ }^{1}$ John Gilligan ${ }^{1}$ \\ Rima Dilbarova $^{1}$ | Kelly Whittaker ${ }^{2}$ Andrew Kossenkov ${ }^{3}$ | \\ George C. Prendergast $^{1,4,5}$ | James M. Mullin ${ }^{1,6}$
}

${ }^{1}$ Lankenau Institute for Medical Research, Wynnewood, Pennsylvania

${ }^{2}$ RayBiotech, Norcross, Georgia

${ }^{3}$ The Wistar Institute, Philadelphia, Pennsylvania

${ }^{4}$ Department of Pathology, Anatomy, and Cell Biology, Sidney Kimmel Medical School, Thomas Jefferson University, Philadelphia, Pennsylvania

${ }^{5}$ Kimmel Cancer Center, Thomas Jefferson University, Philadelphia, Pennsylvania

${ }^{6}$ Division of Gastroenterology, Lankenau Medical Center, Wynnewood, Pennsylvania

\section{Correspondence}

Sunil Thomas, Lankenau Institute for Medical Research, 100 Lancaster Avenue, Wynnewood, PA 19096. Email: thomass-02@mlhs.org; suntom2@gmail.com

\section{Funding information}

Janssen Research Foundation; Wawa Foundation; Women's Board of Lankenau

\begin{abstract}
Patients afflicted with ulcerative colitis (UC) are at increased risk of colorectal cancer. While its causes are not fully understood, UC is associated with defects in colonic epithelial barriers that sustain inflammation of the colon mucosa caused by recruitment of lymphocytes and neutrophils into the lamina propria. Based on genetic evidence that attenuation of the bridging integrator 1 (Bin1) gene can limit UC pathogenicity in animals, we have explored Bin1 targeting as a therapeutic option. Early feasibility studies in the dextran sodium sulfate mouse model of experimental colitis showed that administration of a cell-penetrating Bin1 monoclonal antibody (Bin1 mAb 99D) could prevent lesion formation in the colon mucosa in part by preventing rupture of lymphoid follicles. In vivo administration of Bin $1 \mathrm{mAb}$ altered tight junction protein expression and cecal barrier function. Strikingly, electrophysiology studies in organ cultures showed that Bin $1 \mathrm{mAb}$ could elevate resistance and lower ${ }^{14} \mathrm{C}$ mannitol leakage across the cecal mucosa, consistent with a direct strengthening of colonic barrier function. Transcriptomic analyses of colitis tissues highlighted altered expression of genes involved in circadian rhythm, lipid metabolism, and inflammation, with a correction of the alterations by Bin $1 \mathrm{mAb}$ treatment to patterns characteristic of normal tissues. Overall, our results suggest that Bin $1 \mathrm{mAb}$ protects against UC by directly improving colonic epithelial barrier function to limit gene expression and cytokine programs associated with colonic inflammation.
\end{abstract}

\section{K E Y W O R D S}

circadian rhythm, inflammatory bowel disease (IBD), immunotherapy, inflammation, tight junctions, ulcerative colitis (UC), Bin1 monoclonal antibody 


\section{INTRODUCTION}

Ulcerative colitis (UC) is a chronic, idiopathic inflammatory disease that affects the colon, most commonly afflicting adults aged 30 to 40 years. Patients with UC have mucosal inflammation starting in the rectum that often extends continuously to proximal segments of the colon. UC usually presents with bloody diarrhea and is diagnosed by colonoscopy and histological findings. ${ }^{1}$

People with UC have a higher risk of developing colorectal cancer. ${ }^{2}$ The colorectal cancer risk is as high as $18 \%$ with 30 years of UC. The increased risk of colorectal cancer is due in part to the inflammationinduced loss of barrier function. As yet there are no fully effective drugs or therapies to protect against UC. The inflammatory cytokines, tumor necrosis factor (TNF)- $\alpha$, and IFN- $\gamma$ are elevated in UC. While antiinflammatory drugs and immune suppressors (eg, TNF- $\alpha$ inhibitors) are currently prescribed for UC treatment, side effects such as the risk of opportunistic infections and the lack of efficacy in certain individuals limit the quality of treatment. Hence, there is an interest in developing additional immunotherapies to provide protection against UC. ${ }^{3}$

The bridging integrator 1 (Bin1), also known as amphiphysin 2, is a nucleocytoplasmic adaptor protein with 10 isoforms. BIN1 was initially identified in 1996 as a tumor suppressor; subsequently, however, additional functions have been attributed to different protein transcripts. Bin1 is a conserved member of the BAR (Bin-Amphiphysin-Rvs) family of adapter proteins implicated in diverse cellular processes including endocytosis, actin organization, programmed cell death, stress responses, and transcriptional control. ${ }^{3}$ The BIN1 gene has recently been identified as the most important risk locus for late-onset Alzheimer's disease, after apolipoprotein E. ${ }^{4}$

In a recent study, our group demonstrated that genetic attenuation of Bin1 reduced disease severity in a mouse model of experimental colitis occurring in association with an enhancement of epithelial barrier function. ${ }^{5}$ On the basis of that study, we explored the ability of Bin1 monoclonal antibodies (mAb) developed by our group to phenocopy effects of genetic attenuation in the colitis model. We recently reported the development of a novel colitis therapy targeting the Bin1 protein and supporting epithelial barrier function $^{3}$. We used both cell culture and animal models for the study. In this article, we report progress in the identification of the mechanism by which Bin $1 \mathrm{mAb}$ provides protection in UC.

\section{MATERIALS AND METHODS}

\section{$2.1 \quad$ Bin1 mAb}

Two Bin $1 \mathrm{mAb}$ investigated in this study that was developed in our laboratory previously are designated 99D and 2F11. The antibody 99D recognizes an epitope within the C-terminal Myc binding domain encoded by exon 13, whereas antibody $2 \mathrm{~F} 11$ recognizes an epitope within the $\mathrm{N}$ terminal BAR domain encoded by exons 7 and 8. Although both antibodies specifically recognize Bin1 isoforms, only 99D was found to exert therapeutic activity in applications to reduce colitis morbidity in mice. 99D also exhibited the ability to improve barrier function as demonstrated by in vitro experiments with human $\mathrm{Caco} 2$ colon cells. $2 \mathrm{~F} 11$ was used for western blot analyses in both settings. ${ }^{3}$

\subsection{Experimental colitis model system}

For the study, we used a therapeutic animal model of UC. Animals were weighed before, during, and after treatments. Male mice (C57BL/6) of 5 weeks of age were fed with 3\% dextran sodium sulfate (DSS; Alfa Aesar; MW $40 \mathrm{kDa}$ ) in drinking water ad libitum ( $\mathrm{n}=5$ per group). After 7 days of DSS treatment, mice were provided with distilled water. Twenty-four hours after feeding with water they were injected ip $(0.5 \mathrm{mg}$ of purified antibody per mouse) with Bin $1 \mathrm{mAb}$ or antibody isotype control. ${ }^{3}$ Animals on the long-term study were injected with a second dose of the Bin $1 \mathrm{mAb}$ or antibody isotype control 14 days after the first dose. After 7, 14, and 28 days of Bin1 mAb or isotype control treatments, mice were euthanized and colons were measured and inspected for necropsy for gross macroscopic lesions and used to determine the protein levels by western blot analysis.

The animal studies were approved by the Institutional Care and Use Committee of the Lankenau Institute for Medical Research and performed in accordance with their guidelines under an approved animal protocol.

\subsection{Electrophysiology and epithelial permeability measurements}

Evaluation of transepithelial electrical resistance (TER) and transepithelial mannitol flux was done as described previously. ${ }^{6}$ The mouse cecum was used for analysis in this study due to its larger size facilitating Ussing chamber studies. At necropsy, the cecum was isolated by dissection, sliced longitudinally, and cleaned by ice-cold $\mathrm{pH} 7.35$ bicarbonate buffered saline (Krebs-Ringer bicarbonate, $\mathrm{KRB}$ ). This tissue was then laid on its mucosal surface and the serosal membrane and muscularis propria were surgi- 


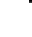

cally removed. Remaining tissue was mounted on $1.13 \mathrm{~cm}^{2}$ Ussing chamber and the tissue bathed in $37^{\circ} \mathrm{C} \mathrm{KRB}$ stirred by gas-lift $\left(95 \% \mathrm{O}_{2}, 5 \% \mathrm{CO}_{2}\right)$ oxygenation. This chamber permits the separation and sampling of luminal versus antiluminal fluid compartments, thereby allowing the precise study of transport and barrier function by the epithelium. TER and transepithelial voltage were measured every 2 minutes using $\mathrm{Ag} / \mathrm{AgCl}$ electrodes in series with $1 \mathrm{M} \mathrm{NaCl}$ agar bridges and a standard current/voltage clamp delivering 1 second pulses of $40 \mu \mathrm{A}$ direct current (McGrath Research and Technology, Phoenix, AZ). After 30 to 45 minutes of incubation, the mucosal tissue was physiologically stabilized and the maximal TER was measured (greater TER values indicate lower electrolyte permeability). Short-circuit current and potential difference measurements were also performed using this apparatus. ${ }^{14} \mathrm{C}$-mannitol (Perkin-Elmer, Boston, MA) along with nonradiolabeled mannitol was then added to the mucosal Hemi chamber (final activity $0.1 \mu \mathrm{Ci} / \mathrm{ml}$, final concentration $0.1 \mathrm{mM}$ ). Aliquots from the serosal fluid Hemi chamber were taken every 15 minutes for 60 minutes for liquid scintillation counting. From linear regression of a graph of cpm per unit time, and using the measured specific activity of the nonmetabolizable ${ }^{14} \mathrm{C}$-mannitol in the mucosal Hemi chamber, we calculated the transepithelial probe flux rate (pmol $\mathrm{min}^{-1} \mathrm{~cm}^{-2}$ tissue). This value reflects the permeability of the TJs to D-mannitol. ${ }^{6}$

\subsection{Western blot analysis}

Bin1 mAb treated or control colons were dissected into upper and lower colon. Mucosal tissue was physically scraped and lysed as previously described. ${ }^{7}$ After polyacrylamide gel electrophoresis $(10 \%$ gel) and transfer to nitrocellulose membrane, they were probed with antibodies for claudin proteins (Claudin-2, -3, and -5; 1-2 $\mu \mathrm{g} \mathrm{mL}^{-1}$; Life Technologies, CA) according to the manufacturer's instructions.

\subsection{RNA-seq analysis}

Twelve RNA samples from four groups, control (ctr), DSS treated, IgG, or Bin1 antibody treated, were first treated with Invitrogen/Ambion DNA-free kit (cat \#AM1906) to remove DNA and run on the Agilent 2100 Bioanalyzer for quality assessment. The sequencing libraries were generated using the Lexogen QuantSeq 3' mRNA sequencing library preparation kit (SKU:015.24) for Illumina sequencing platform compatible libraries. The KAPA real-time PCR Library Quantification Kit (KK4835) was used to quantify and assess library quality. All libraries were pooled in equal molar amounts and run on an Illumina NextSeq 500 using the Mid Output V2 (150 cycles) kit (cat \# FC-404-2001) in a 75 base pair single read run. Raw reads were aligned using bowtie 2 algorithm ${ }^{7}$ against mm10 genome, and Ensembl transcrip- tome information was used along with RSEM software ${ }^{8}$ to calculate raw read counts for each gene. Differential expression analysis was done using DESeq2. ${ }^{9}$ Genes significantly affected by DSS treatment (false discovery rate $[\mathrm{FDR}]<10 \%)$ as compared with controls were considered and genes with expression significantly rescued by Bin1 over IgG antibody $(P<0.05)$ were taken for enrichment analysis. Expression data for top changed genes were visualized in a heatmap using Microsoft Excel. Analysis of the gene set for the enrichment of Upstream Regulators was done using QIAGEN's Ingenuity Pathway Analysis software (IPA, QIAGEN Redwood City; www.qiagen.com/ingenuity). Only regulators with targets significantly enriched at least $P<10^{-7}$ and activation state predicted with Z-score of at least 1 were reported.

\subsection{Antibody array}

Male mice (C57BL/6) of 5 weeks of age were fed with $3 \%$ DSS (Alfa Aesar, MW $40 \mathrm{kDa}$ ) in drinking water ad libitum ( $n=2-3$ per group). After 7 days of DSS treatment, mice were provided with distilled water. Twenty-four hours after feeding with water they were injected ip ( $0.5 \mathrm{mg}$ per mouse) with Bin $1 \mathrm{mAb}$ or antibody isotype control. Mice were euthanized and colons were measured and inspected for necropsy for gross macroscopic lesions on day 7 after injection with Bin1 mAb or isotype control. Tissues were then homogenized in RIPA buffer and protein concentration was quantified with a BCA protein assay kit (Pierce). The concentration of 40 mouse cytokines and chemokines was analyzed with Mouse Quantibody Cytokine Arrays Q5 (QAM-CYT-4000; RayBiotech) by the RayBiotech testing service, according to manufacturer's instructions. Briefly, samples were incubated with slides preprinted with the 40 respective capture antibodies. A standard mix, consisting of known amounts of recombinant proteins representing all 40 proteins were used to quantify the concentration of every cytokine. Sample incubation was followed by extensive washing to remove any nonbound proteins in the sample matrix. Next, a biotinylated detection antibody cocktail was incubated on the array. After washing away unbound biotinylated detection antibodies, the arrays were incubated with a fluor-conjugated with streptavidin and the fluorescent signals were visualized using a fluorescent scanner (InnoScan 710; InOpsys ). Inter- and intra-slide normalization of fluorescent signal intensities was conducted using positive controls consisting of biotin-labeled IgG, and negative controls, consisting of buffer only. Average signal intensities of quadruplicate spots, minus outliers, were used for all calculations. Outliers were determined as values over $30 \%$ above the average signal intensity 


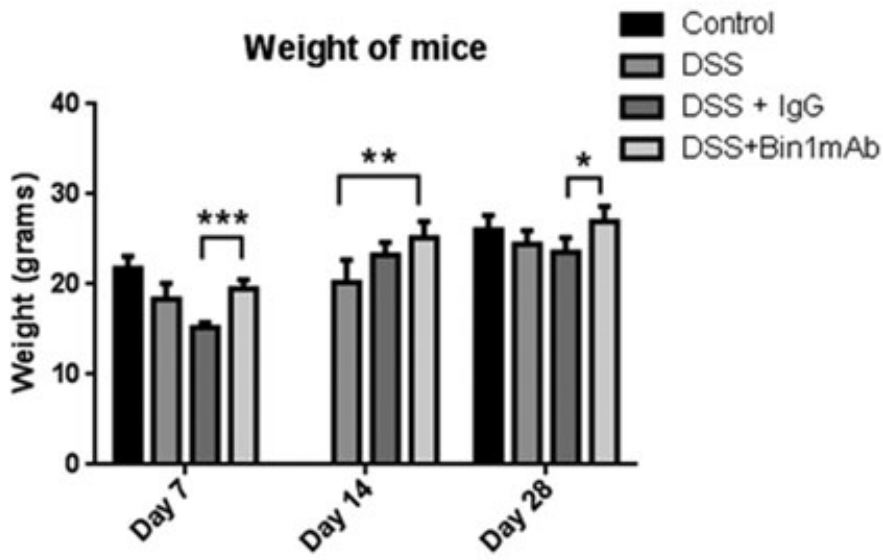

FIG URE 1 Bin1 antibody treatment altered the weight of mice. There was a change in weight of mice treated with Bin1 $\mathrm{mAb}$ after subjecting to DSS treatment of 7 days $\left({ }^{*} P<0.05,{ }^{* *} P<0.01\right.$, ${ }^{* * * *} P<0.001$ as determined by $t$ test). Bin 1 , bridging integrator 1; DSS, dextran sodium sulfate; mAb, monoclonal antibodies

across the quadruplicate spots. Data below the lower limit of detection were discarded.

\subsection{Immunohistochemistry}

Caco2 BBE cells (passages 55-63), an epithelial cell line derived from human colon carcinoma, were obtained from American Type Culture Collection (CRL- 2102). The method of Wang et $\mathrm{al}^{10}$ was followed for culture and maintenance of cells. Sixty percent confluent cells were treated with Bin1 $\mathrm{mAb}$ or isotype control for 48 hours and the cells stained following the method of Rybakovsky et al. ${ }^{11}$ The cells were probed with the primary antibodies: EEA1, Beclin-1, Sequestosome1/p62, CD32, and ubiquitinylated antibodies (1:500; Thermo Fisher Scientific, MA); followed by the secondary antibodies anti-rabbit Alexa 488/592, anti-mouse Cy3 (1:500) and DAPI. The cells were mounted using Fluoromount-G (Southern Biotech, AL) and visualized by confocal microscopy (Nikon Eclipse TI, Japan). Images were taken from different fields from the same slide. The experiments were repeated three times.

\section{8 $\quad$ Statistical analyses}

Unpaired two-tailed Student $t$ tests were used to compare sets of data obtained from independent groups of animals. Statistical significance was considered at the $P<0.05$ level.

\section{RESULTS}

\section{1 | Bin1 mAb 99D protects colon mucosa in an animal model of UC}

DSS-induced colitis is commonly used to address the pathogenesis of inflammatory bowel disease (IBD). We demonstrated recently that the Bin 1 mAb (99D) could provide protection in the DSS murine model of UC. ${ }^{3} \mathrm{We}$ determined previously that a single administration of $0.5 \mathrm{mg}$ Bin $1 \mathrm{mAb}$ was the optimum dosage to prevent colitis. Previously, ${ }^{3}$ we reported on the histology of the colon during Bin $1 \mathrm{mAb}$ treatment after the induction of colitis; hence, they are not reported in this article. In this article, we further investigated the biological effects and mechanism of action of the therapeutic effect. In the design used, mice were administered with $3 \%$ DSS in

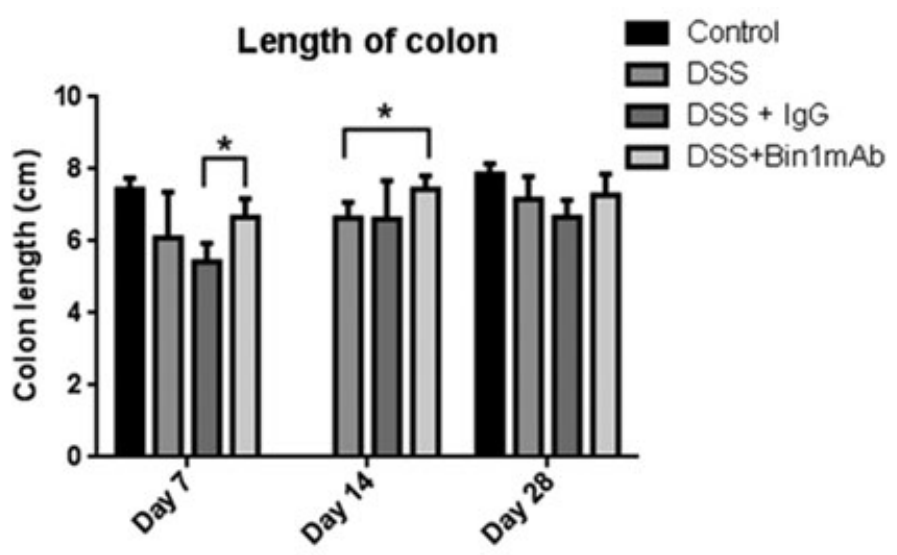

FIG URE 2 Bin1 antibody treatment altered the length of the colon. There was a change in length of the colon of mice treated with Bin $1 \mathrm{mAb}$ after subjecting to DSS treatment of 7 days. $\left({ }^{*} P<0.05\right.$ as determined by $\mathrm{t}$ test). Bin1, bridging integrator 1; DSS, dextran sodium sulfate; $\mathrm{mAb}$, monoclonal antibodies 
(A)

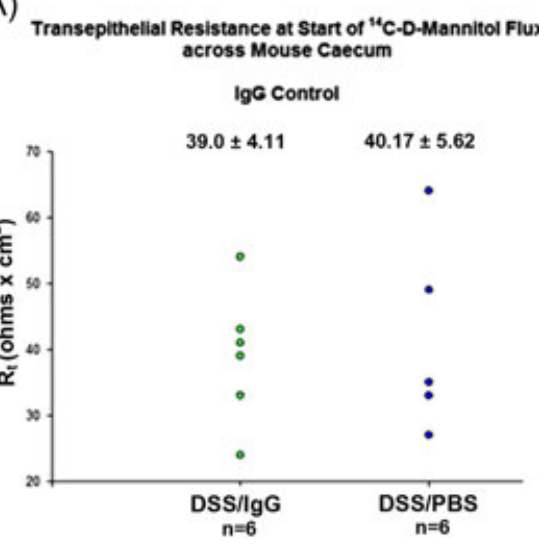

(B)

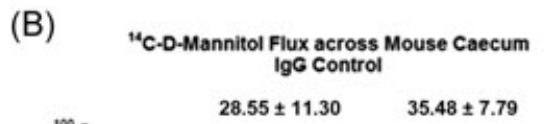

FIG URE 3 Colon of mice treated with Bin1 mAb had A, high resistance and $\mathrm{B}$, low flux compared to the controls. Bin1, bridging integrator $1 ; \mathrm{mAb}$, monoclonal antibodies
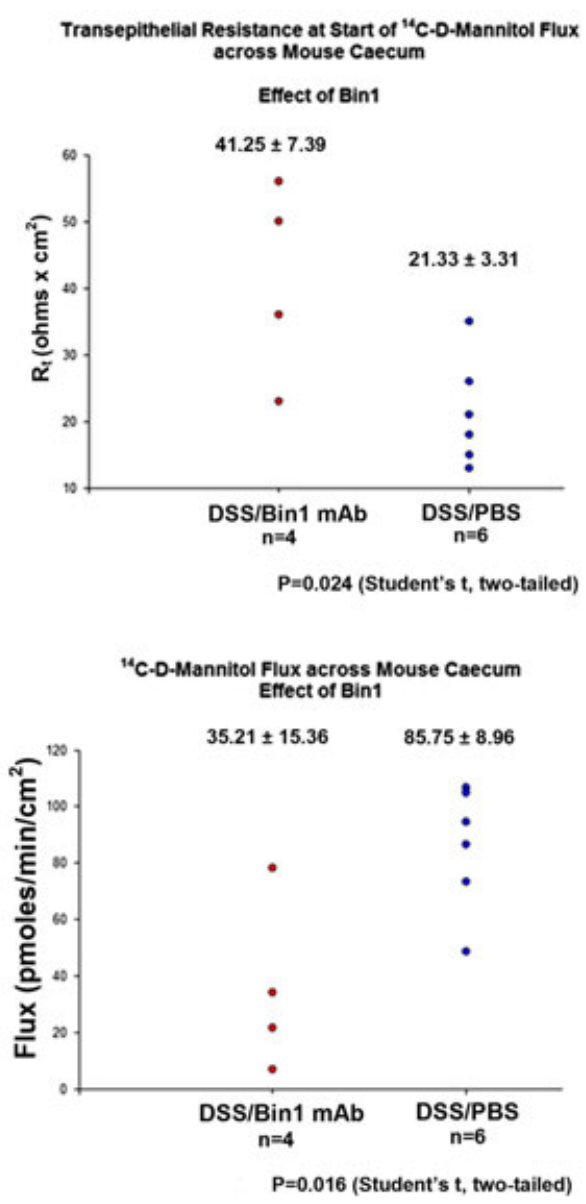

water for 7 days (standard protocol) followed by providing distilled water. Animals develop colitis within 7 days of treatment with DSS. Twenty-four hours after feeding with water the animals were injected with $0.5 \mathrm{mg}$ of Bin $1 \mathrm{mAb}$ or nonspecific murine IgG (isotype-matched to 99D). The other controls included mice that were untreated or DSS-only treated mice. We performed both short-term and long-term treatments. Short-term studies involved treating DSS exposed animals with Bin1 $\mathrm{mAb}$ for 7 or 14 days. In long-term studies, animals exposed to DSS were given two doses of Bin $1 \mathrm{mAb}$ that were 14 days apart, with the animals euthanized on day 28 after the first dose.

Mice treated with Bin $1 \mathrm{mAb}$ did not lose weight after exposure to DSS. The Bin $1 \mathrm{mAb}$ treated animals exhibited greater weight on days 7, 14 and 28 compared to control subjects that were untreated or treated with IgG (Figure 1). As expected, DSS-induced erosion of the colon tissue structure and thereby impacted the physiology and metabolism of the animals. However, in protecting animals from colitis, Bin $1 \mathrm{mAb}$ administration specifically prevented the characteristic weight loss induced by DSS treatment.
In animals exposed to DSS there is a significant decrease in colon length. ${ }^{5}$ Hence, colon length measurement has been used as a morphological parameter of the degree of tissue damage in DSS colitis. ${ }^{12}$ In our study, mice from each group were euthanized on days 7, 14, and 28 post $\mathrm{mAb}$ treatment and the colon dissected and measured. Mice treated with Bin $1 \mathrm{mAb}$ had significantly longer colons on days 7 and 14 compared with the IgGtreated or DSS alone controls (Figure 2). In contrast, there was no significant change in the length of colons isolated from mice up to 28 days after Bin1 $\mathrm{mAb}$ treatment, consistent with a mitigating effect of the treatment on the manifestation of colitis in the model.

\section{2 | Bin1 mAb treatment improves basal intestinal barrier function}

To investigate the effects of targeting of Bin1 with the 99D antibody on intestinal epithelial cell barrier function, we used an established organ culture system using murine cecal colon segments. ${ }^{13}$ Specifically, cecal segments from mice were dissected and mounted in Ussing chambers to measure mucosal barrier function by 
(A)

Distal colon (Day 7)

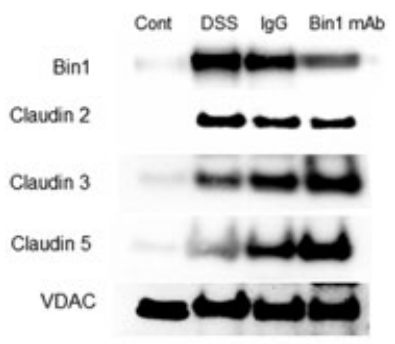

(B)

B) Distal colon (Day 14)

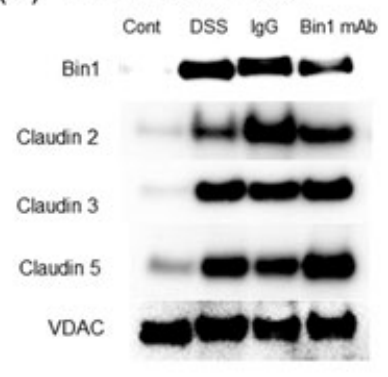

(C)

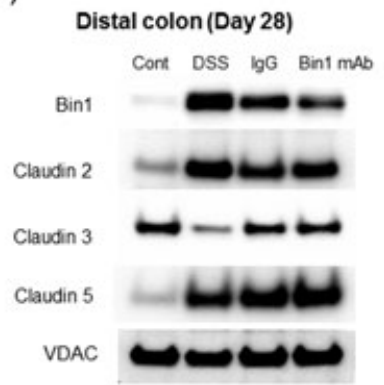

Proximal colon (Day 7)

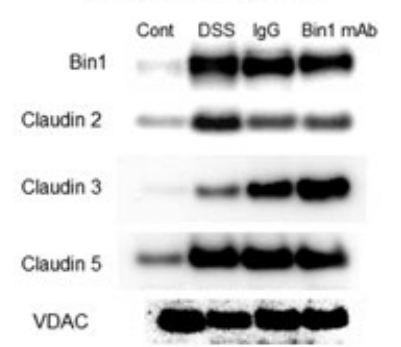

3.3 Bin1 mAb treatment impacts the colon tight junction proteins

In our previous study we used a cell culture model of Caco2 BBe and demonstrated that Bin $1 \mathrm{mAb}$ induced changes in tight junction proteins. In this study, the colon of the euthanized mice was divided into proximal and distal halves. Each was lysed and subjected to western blot analysis. Initially, we probed the levels of the Bin1 protein in the proximal and distal colon using the $2 \mathrm{~F} 11$ Bin1 antibody. There was a low expression of the Bin1 protein in the proximal and distal colon of Bin1 mAb-treated animals compared with the controls. Similarly, the expression of the pore-forming claudin-2 was lower in the Bin $1 \mathrm{mAb}$-treated mice exposed to DSS compared with controls on days 7,14 , and 28 in the proximal as well as distal colons. However, the Bin1 treated mice exposed to DSS had higher expression of claudin- 3 and -5 in both the proximal and distal colons on days 7,14 , and 28 compared with controls. The results demonstrated that Bin $1 \mathrm{mAb}$ increased the expression of the tight junction barrier proteins that may protect against colitis (Figure 4A-C; Supporting Information Figures 1-3).

\subsection{Altered gene expression patterns induced by DSS are corrected by Bin1 mAb treatment}

To determine genes significantly affected by Bin $1 \mathrm{mAb}$, we assayed transcriptome expression changes using RNA-seq. We found 209 genes that were significantly affected in DSS mice vs control (FDR $<10 \%)$ and after 7 days of Bin $1 \mathrm{mAb}$ treatment. The top 32 most significantly changed genes are shown in Figure $5 \mathrm{~A}$. Bin $1 \mathrm{mAb}$ treatment increased the expression of genes negatively related to obesity. Bin $1 \mathrm{mAb}$ treatment upregulated adiponectin, adipsin (complement factor D), carbonic anhydrase 3 (Car3), and thiosulfate sulfurtransferase (TST) involved in obesity, whereas they are decreased during colitis (Figure 5A).

Bin $1 \mathrm{mAb}$ treatment increased the expression of UGDH (involved in regulating inflammation) as well as Sepp1 and Spint2 (involved in inhibiting colon cancer induced during inflammation). The cytochromes are involved in the metabolism of drugs. Bin $1 \mathrm{mAb}$ treatment increased the expression of the genes Cytochrome P450 (Cyp4f16), Sulfotransferase family 1B member 1 (Sult1b) involved in drug metabolism.

Environmental triggers such as sleep disorders could impact disease course in IBD. ${ }^{14}$ Circadian rhythms in gene expression are regulated at the cellular level by a molecular clock comprising a core set of clock genes/proteins. The genes involved in lipid biosynthesis and fatty acid oxidation 
(A)

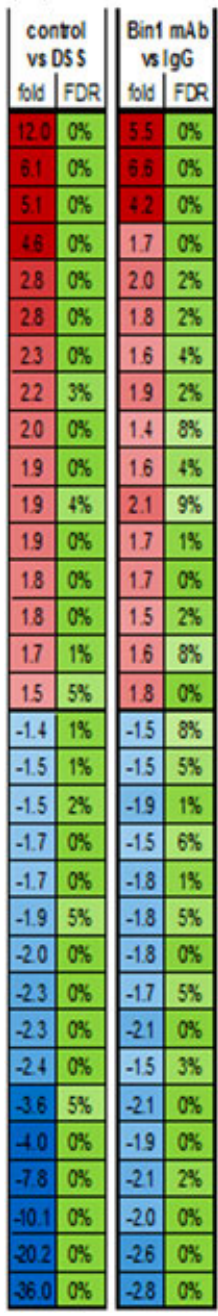

(B)

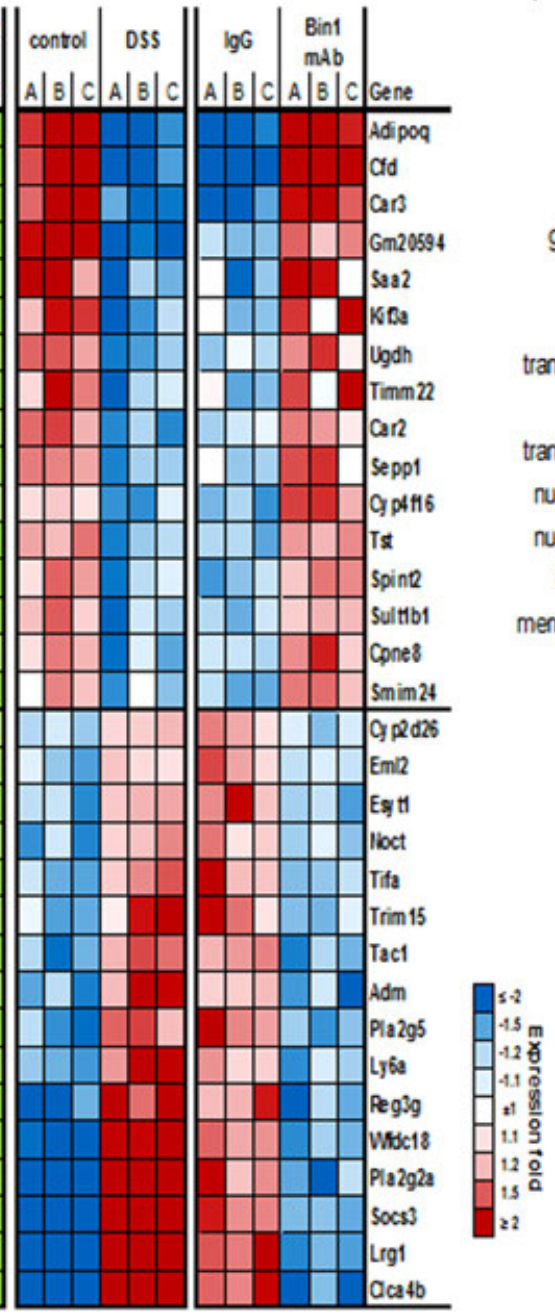

\begin{tabular}{|c|c|c|c|c|}
\hline Type & Regulator & $\mathrm{N}$ & pvalue & 2 \\
\hline cytokine & TNF & 47 & $2 \times 10^{14}$ & -3.4 \\
\hline cytokine & IL 18 & 27 & $2 \times 10^{-9}$ & -26 \\
\hline cytokine & OSM & 19 & $7 \times 10^{8}$ & -2.3 \\
\hline gran th facta & TGFB1 & 42 & $2 \times 10^{11}$ & -2.2 \\
\hline complex & NFKB & 22 & $4 \times 10^{-9}$ & -2.2 \\
\hline cytokine & IFNG & 32 & $1 \times 10^{8}$ & $\mid-1.8$ \\
\hline scription factor & CEPPB & 19 & $7 \times 10^{10}$ & $\mid-1.8$ \\
\hline cytokine & IL.2 & 10 & $4 \times 10^{-9}$ & -1.5 \\
\hline scription factor & CEPPA & \begin{tabular}{|l|}
17 \\
\end{tabular} & $6 \times 10^{8}$ & 1.3 \\
\hline uclea receptar & PPARA & 17 & $7 \times 10^{8}$ & 1.8 \\
\hline clea recepta & PPARG & \begin{tabular}{|l|}
18 \\
\end{tabular} & $4 \times 10^{8}$ & 24 \\
\hline ion channel & CFTR & 14 & $6 \times 10^{15}$ & 26 \\
\hline ibane recept & IL 10RA & 23 & $9 \times 10^{18}$ & \\
\hline
\end{tabular}

Type

cytokine

cytokine

gav th tacto

complex

cription bad

nuclea receptor

clea receptor

mbane recepta

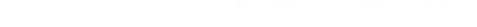

F I G U R E 5 Gene expression analysis results. A, Top genes most significantly affected by DSS (FDR $<10 \%$ ) and significantly rescued by $\mathrm{Bin} 1 \mathrm{mAb}(\mathrm{FDR} 1<0 \%)$. B, Ingenuity Pathway Analysis results with upstream regulators that have a significant number of targets $(\mathrm{N})$ in the list of 209 genes. Activation Z-scores (Z) calculated by IPA based on targets mRNA changes predicts the activity of regulatory proteins.

Bin1, bridging integrator 1; DSS, dextran sodium sulfate; FDR, false discovery rate

are rhythmically activated and repressed by clock proteins, hence providing a direct mechanism for circadian regulation of lipids. DSS-induced colitis leads to the increase in the expression of genes involved in circadian rhythm (Noct/ nocturnin and cyp2d26/ Cytochrome P450 2D26) and sphingolipid metabolism (ESYT1/Extended Synaptotagmin 1); whereas, Bin1 mAb treatment lowered their expression.

Bin1 mAb treatment lowered the expression of genes involved in inducing inflammation including, Tifa, Trim15, Tac1, Adm, Socs3, Ly6a, Lrg1, and Clca4b. Bin1 $\mathrm{mAb}$ treatment also lowered the expression of regenerating family member 3 gamma and Pla2g2a. REGs and Pla2g2a are highly expressed during IBD. ${ }^{15,16}$

We also analyzed the list of 209 genes to determine the genes altered by the treatment with Bin1 mAb. Using Ingenuity Pathway Analysis ${ }^{17-19}$ we found five regulators
(Figure 5B) with a significant number of known targets in the list and whose activity was predicted to be activated upon Bin1 mAb treatment. Of the eight regulators predicted to be inhibited by Bin $1 \mathrm{mAb}$, five were cytokines, including TNF, IL-1B, IFN- $\gamma$, OSM, and IL22. Bin1 mAb activates PPARA, PPARG, and IL-10RA known to have anti-inflammatory effects.

\section{5 | Bin1 mAb treatment reduces the expression of inflammatory cytokines}

Currently, TNF-inhibitors are used in the treatment of UC. These inhibitors are known to lower the expression of inflammatory cytokines. We determined the expression of the cytokines using RayBiotech antibody arrays. Bin1 mAb lowered the expression of inflammatory cytokines including 


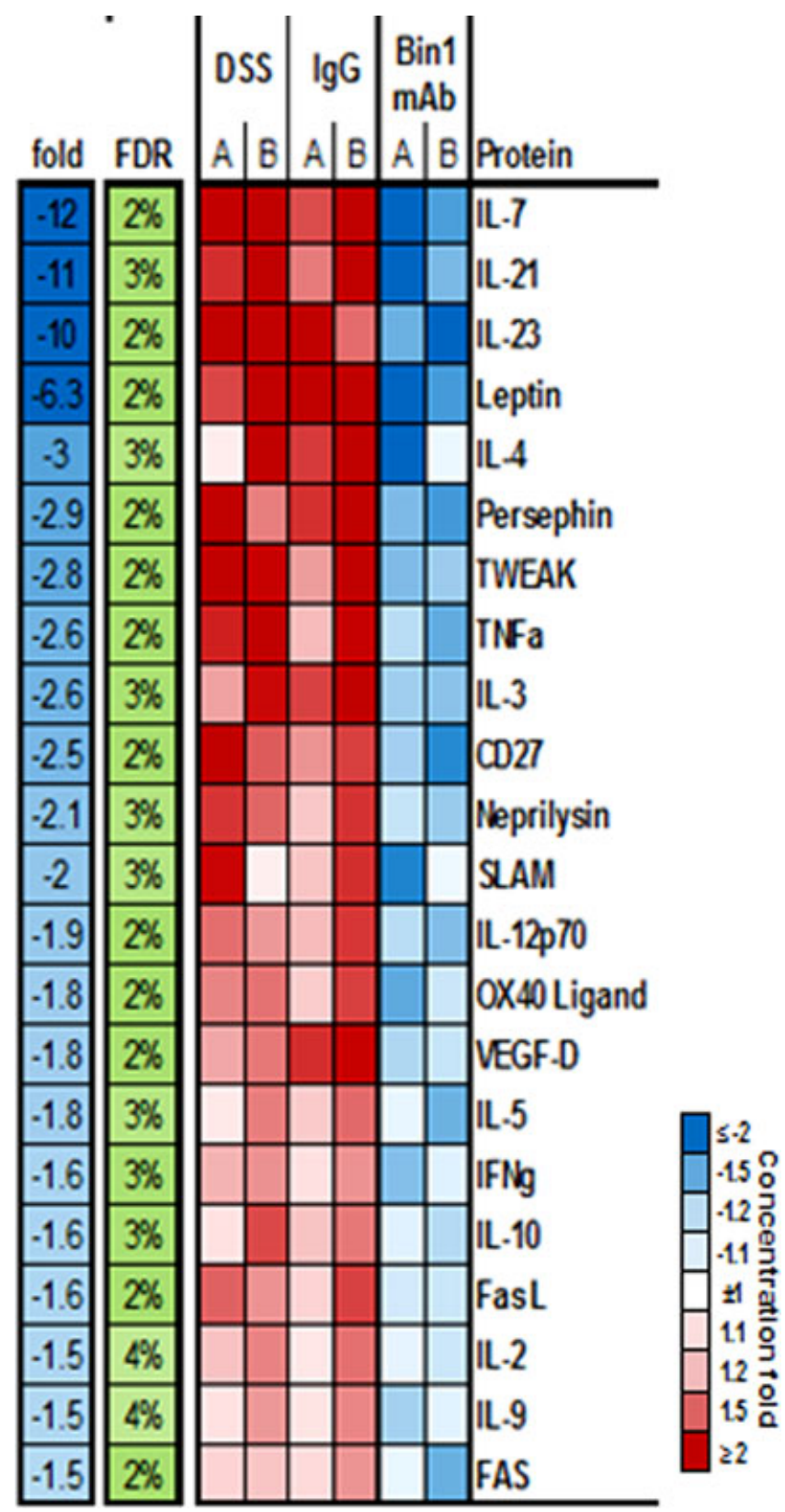

F I G U RE 6 Antibody array analysis data. Proteins significantly reduced by $\operatorname{Bin} 1 \mathrm{mAb}(\mathrm{FDR}<5 \%)$. The difference of concentration of proteins between Bin $1 \mathrm{mAb}$ and all other groups was tested using two-tail two-sample $t$ test and nominal $P$ values were corrected for multiple testing using the Benjamini-Hochberg method. Proteins that were significantly reduced with FDR $<5 \%$ were considered. Bin1, bridging integrator $1 ; \mathrm{mAb}$, monoclonal antibodies; FDR, false discovery rate

TNF- $\alpha$, TWEAK (TNF family), and IFN- $\gamma$. The expression of the cytokines, IL-2, -3, -4, -5, -7, -9, -10, -21, -23, and IL-12p70 was also decreased on treatment with the Bin $1 \mathrm{mAb}$. These cytokines are known to be inducers of UC and other IBD diseases. The expression of the T-cell co-stimulatory molecules CD27 and OX-40L, as well as the expression of the monocytes/macrophages co-stimulatory molecule SLAM, is reduced on treatment with the Bin1 mAb. The apoptosis molecules Fas and FasL are upregulated in UC. However, Bin1 mAb treatment led to the lower expression of these molecules after the induction of DSS-induced colitis in mice (Figure 6).

\section{6 | Bin1 mAb is taken into cells by multiple mechanisms}

Bin1 is a protein localized on the membrane and in the cytosol. In our study, Bin1 mAb efficacy assumes intracellular access of the $\mathrm{Ab}$. It is however clearly not understood how the Bin $1 \mathrm{mAb}$ is taken into cells. In this study and our previous study, ${ }^{3}$ we demonstrated that Bin $1 \mathrm{mAb}$ could lower the expression of Bin1 protein in colon epithelial cells. To determine how the Bin $1 \mathrm{mAb}$ is taken into cells we incubated $\mathrm{Caco} 2$ cells in the presence of Bin $1 \mathrm{mAb}$ and analyzed by confocal microscopy how they are taken into cells.

Endosomes are involved in the transport of nutrients as well as microorganisms into cells. ${ }^{20}$ Recently, we observed high expression of the endosomal proteins EEA1 and Beclin-1 in the mucosal and muscularis region of mouse colon. ${ }^{21}$ On the basis of this data we hypothesized that the endosomes in the colon may be involved in compartmentalizing the host microbiome to prevent entry into the tissues. To determine if the endosomes are also observed in the Caco 2 cells, we cultured the cells and observed the expression of the early endosomal protein EEA1 at 70\% confluence. Most of the EEA1 endosomes were localized on the margins of the Caco2 cells (Figure 7A). The Bin $1 \mathrm{mAb}$ was localized to the EEA1 endosomal proteins.

To determine if other endosomal proteins are colocalized with the Bin1 $\mathrm{mAb}$, we probed for Beclin-1 and sequestosome/p62. Analysis of Caco2 cells incubated with Bin1 mAb by confocal microscopy demonstrated colocalization of Beclin-1 and sequestosome/p62 with the Bin $1 \mathrm{mAb}$ supporting that the Bin $1 \mathrm{mAb}$ could be taken into the cells by endosomes (Figure 7B and 7C).

In neuronal cells, $\mathrm{Gu}$ et $\mathrm{al}^{22}$ and Congdon et $\mathrm{al}^{23}$ demonstrated antibody entry mediated by Fc gamma receptor endocytosis. Caco2 cells were incubated in the presence of Bin $1 \mathrm{mAb}$ or isotype controls for 48 hours and probed with $\mathrm{Fc}$ gamma RII (CD32). Caco2 exhibits heterogeneity, with a different subpopulation of cells during cell culture. ${ }^{24}$ We observed that not every cell of the cultured $\mathrm{Caco} 2$ incorporated Fc gamma RII (CD32). Those cells exhibiting Fc gamma RII had Bin1 mAb antibody colocalized with it as observed by the overlap of the antibody and the receptor (Figure 7D). Our studies show that multiple intracellular and extracellular proteins are involved in the uptake of Bin1 mAb into cells, 

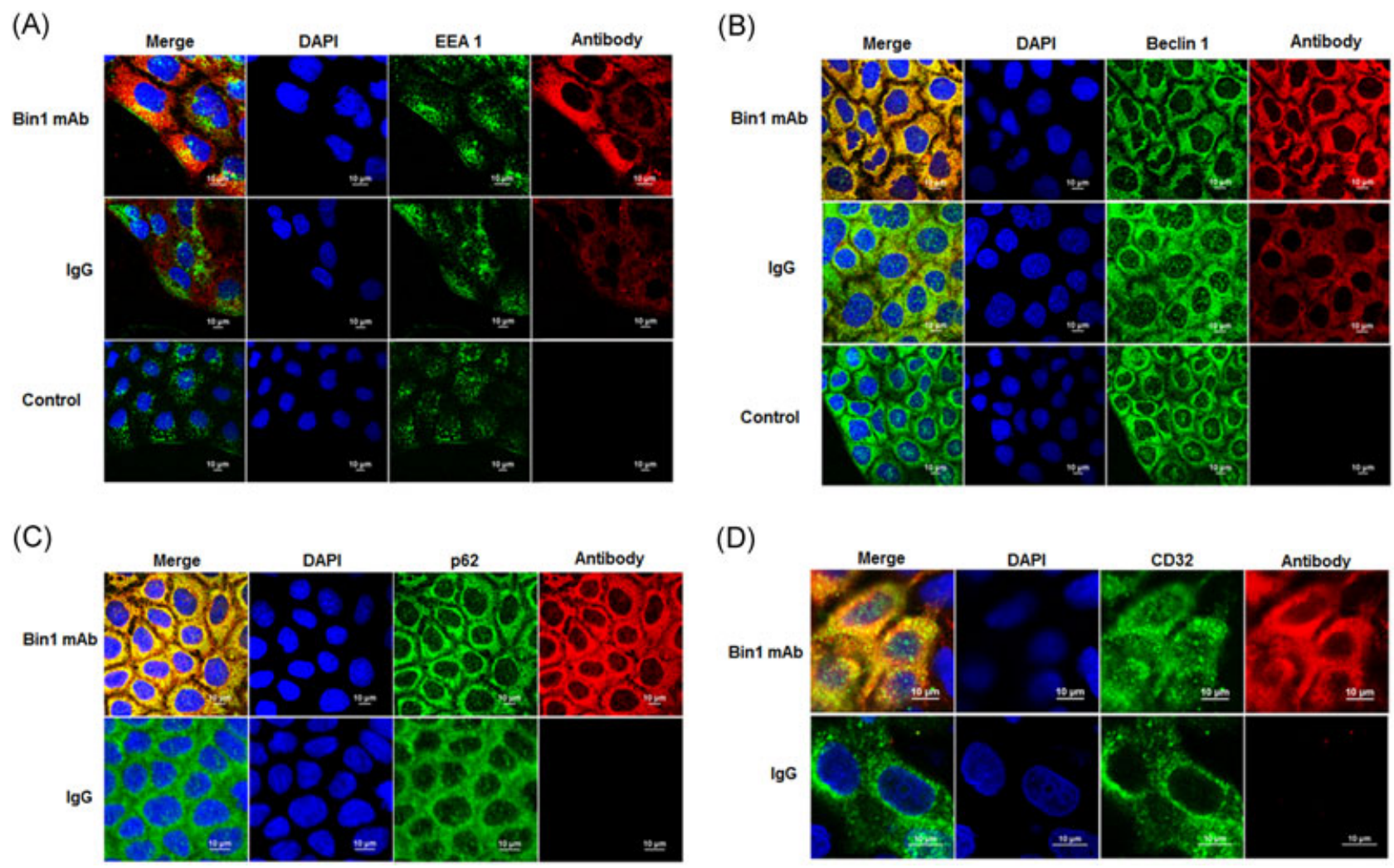

FIG URE 7 Colocalization of Bin1 mAb with endosomal and Fc gamma receptor proteins in Caco2 cells observed by confocal microscopy. Bin1 mAb is colocalized (orange) with A, EEA1; B, Beclin-1; C, p62/sequestosome; and D, CD32 (Fc gamma RII). Bin1, bridging integrator $1 ; \mathrm{mAb}$, monoclonal antibodies

but most important, that Bin1 mAb has access to intracellular targets.

\section{DISCUSSION}

The Bin1 protein is localized in several tissues, including the large intestine. ${ }^{25}$ In a recent article we reported that administration of DSS to mice induced severe lesions throughout the mucosa, rupture of the lymphoid follicle, high-level neutrophil, and lymphocyte infiltration into the mucosal and submucosal areas, and loss of surface crypts; whereas treatment with Bin1 mAb reduced colitis morbidity. In vitro studies in human $\mathrm{Caco} 2$ cells showed that Bin1 antibody altered the expression of tight junction proteins and improved barrier function indicating at least some direct effect on the epithelium. Our data suggested that a therapy based on Bin1 monoclonal antibody supporting mucosal barrier function and protecting the integrity of the lymphoid follicle could offer a novel strategy to treat UC and possibly limit risks of colorectal cancer. However, the mechanism by which Bin $1 \mathrm{mAb}$ protects the animals against colitis is not known. ${ }^{3}$

Bin1 $\mathrm{mAb}$ treatment lowered the expression of the Bin1 protein in the proximal and distal colon (Figure 4A and 4B), demonstrating that the Bin1 mAb was effective in lowering the specific protein levels in the animals. This infers access of the mAb to the cell interior. The colon of the DSS treated mice was shorter and had a bloody stool after 7 days of treatment; whereas, treatment with Bin $1 \mathrm{mAb}$ protected against colitis. There was no blood in the stool and the nature of the colon was similar to the untreated controls. To determine the leakiness of the colon we measured the TER as well as mannitol permeability. Bin1 mAb-treated mice displayed an elevation in basal TER as well as a corresponding decrease in mannitol permeability, both of which directly implicated improvement of barrier function. Tight junctions form the apical-most unit of the epithelial barrier, defining the boundary between the apical and basolateral membranes and are predominantly the rate-limiting factor in the paracellular passage. The tight junction is built up by both transmembrane proteins such as occludin, tricellulin, different claudins, and junctional adhesion molecules, as well as intracellular proteins such as zonula occludens $-1,-2$, -3 and cingulin. ${ }^{26}$ Western blot analysis demonstrated high expression of the tight junction proteins claudins- 3 and -5 after the treatment with $\operatorname{Bin} 1 \mathrm{mAb}$. The expression of the pore-forming claudin-2 is increased in $\mathrm{UC}^{27}$ and the expression is decreased on treatment with the Bin1 $\mathrm{mAb}$ (Figure 4A and 4B). 
The incidence of UC is highest in the western hemisphere ${ }^{28}$; however, recent studies show that the incidence of UC is increasing in developing countries. ${ }^{29,30}$ The Western diet pattern is dominated by increased consumption of refined sugar, omega- 6 polyunsaturated fats, and fast food, combined with a diet deficient in fruit, vegetables, and fiber. This shift to the Western diet pattern is hypothesized to increase proinflammatory cytokines, modulate intestinal permeability, and alter the intestinal microbiota promoting a low-grade chronic inflammation in the gut.

Lipids have emerged as important components of cellular signal transduction pathways. Their roles in modulating host inflammatory responses are of clinical interest as agents of both promotion and resolution. Dietary lipids are implicated in the pathogenesis of chronic intestinal inflammation, including IBD. Perturbations in lipid metabolism could contribute to the development of IBD. ${ }^{31,32}$ Biologically active sphingolipids, such as sphingosine-1-phosphate (S1P) are important regulators of lipid metabolism. S1P is derived from the recycling of endogenous human sphingolipids and the metabolism of dietary animal products containing sphingolipids. S1P signals through a family of five $G$ protein-coupled receptors. In addition, it activates nuclear factor kappa $B$ (NF- $\mathrm{kB}$ ) and signals transducer and activator of transcription 3 , two transcriptional regulators that serve as master switches in inflammation and carcinogenesis. Through these and other mechanisms, a causal role for S1P in inflammatory conditions including UC and colorectal cancer has been implicated. $^{32,33}$ Phospholipase A2 (PLA2) is a group of enzymes that hydrolyze phospholipids to liberate fatty acids and lysophospholipids, representing the first rate-limiting step in the biosynthesis of a variety of lipid mediators. UC involves the synthesis of eicosanoids from arachidonic acid, which is released from membrane phospholipids by PLA2. ${ }^{34}$ Clinical and biochemical evidence suggests that secreted PLA2 group III (sPLA2-III) is associated with colorectal cancer. PLA2 KO mice are less susceptible to DSS-induced colitis and are highly resistant to colon carcinogenesis implying that the amelioration of colonic inflammation by sPLA2-III ablation may underlie the protective effect against colon cancer. ${ }^{34}$ Our data show that Bin1 mAb treatment reduces the expression of PLA2, whereas untreated animals with colitis had high levels of PLA2 (see Figure 5A).

In IBD, obesity is associated with worsening of the course of the disease. Obesity is a complex disease involving dysbiosis of the gut microbiota, lipid accumulation, and insulin resistance. ${ }^{35}$ Obesity, adipose inflammation, and malfunction of $\beta$ cells are tightly associated with one another, but the molecular link remains to be established. ${ }^{36}$ Increasing adiposity is directly correlated with adipose inflammation and elaboration of proinflammatory cytokines such as TNF- $\alpha .{ }^{37}$ Until recently, the role of fat in the development of obesity was considered to be a passive one.
Adipocytes were considered storage cells for fat. However, a current view is that adipocytes are a critical component of metabolic control and function as endocrine organs that have both good and bad effects. Small adipocytes in lean individuals promote metabolic homeostasis; the enlarged adipocytes of obese individuals recruit macrophages and promote inflammation and the release of a range of factors that predispose toward insulin resistance. ${ }^{38}$ The proteins and cytokines that regulate the adipose tissue may be pro- or antiinflammatory. Car3 is an abundant protein in adipose tissue and it decreases with obesity. ${ }^{39}$ Similarly, transgenic overexpression of TST in adipocytes protected mice from dietinduced obesity and insulin-resistant diabetes. TST-deficient mice showed markedly exacerbated diabetes, whereas pharmacological activation of TST ameliorated diabetes in mice. $^{40}$ Our RNA array data show that DSS exposure decreases the expression of the genes involved in obesity; whereas, Bin1 mAb treatment upregulates the gene expression (Car3, TST).

Several studies support associations among sleep, immune function, and inflammation. ${ }^{41}$ As yet it is not known whether lack of sleep contributes to IBD. Recently, there has been an increased interest in the roles that sleep, circadian rhythms, and melatonin could have as regulators of inflammation in the GI tract. Advances in our understanding of the molecular machinery of the circadian clock and the discovery of clock genes in the GI tract are opening up new avenues of research for a role of sleep in IBD. Altering circadian rhythm significantly worsens the development of colitis in animal models, and preliminary human studies have shown that patients with IBD are at increased risk for altered sleep patterns. ${ }^{42}$ A recent study demonstrated that melatonin may be clinically applicable for patients with IBD, particularly those suffering from sleep disturbances. ${ }^{43}$

Our studies show that UC could lead to changes in the genes of the circadian clock. The genes involved in the circadian rhythm (Nocturnin, Cytochrome P450 2D26) are altered by Bin $1 \mathrm{mAb}$ treatment demonstrating that the antibody could influence these genes (see Figure 5A). The circadian timing system plays a key role in regulating lipid metabolism. The circadian system ensures that daily rhythms in lipid absorption, storage, and transport are temporally coordinated with rest activity and feeding cycles. At the cellular level, genes involved in lipid synthesis and fatty acid oxidation are rhythmically activated and repressed by core clock proteins. In mammals, the core clock components are CLOCK, BMAL, PER, and CRY. ${ }^{44}$ Mice with a mutated CLOCK gene were prone to develop hypertriglyceridemia. A key role in the regulation of lipid metabolism was determined for Nocturnin, a gene expressed in a circadian manner, whose product is an enzyme with a deadenylase activity. Nocturnin-deficient mice on a high-fat diet did not increase their body weight and visceral fat and did not 


\section{(A)}

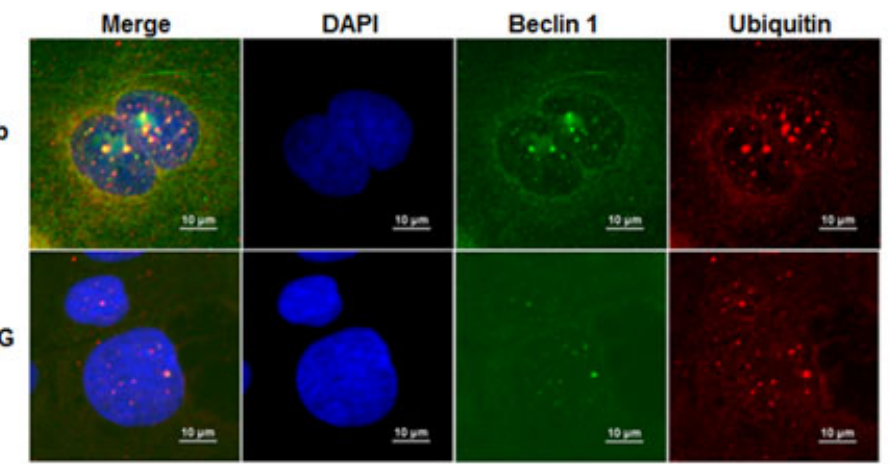

(B)

(C)
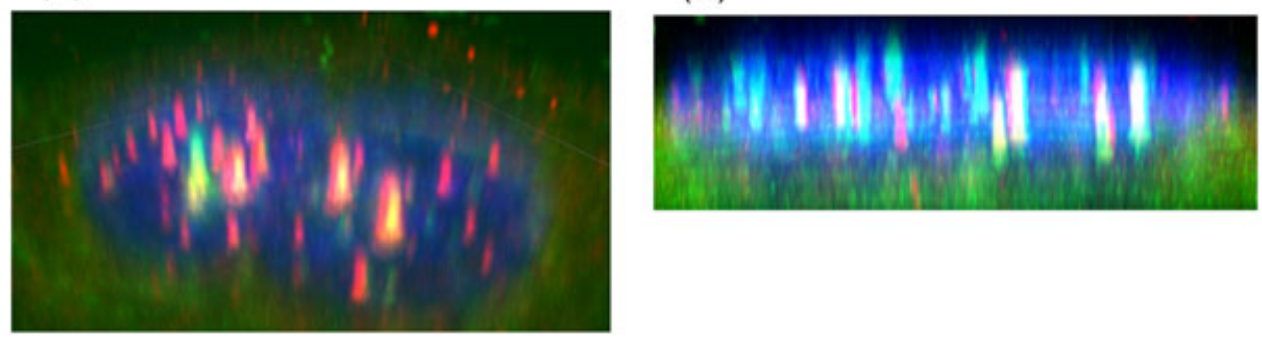

F I G U RE 8 Expression of ubiquitination in the cytoplasm and nucleus of Caco2 cells by confocal microscopy after incubation with Bin1 mAb. A, Caco 2 cells treated with Bin 1 mAb shows ubiquitination in the cytoplasm and nucleus. The Beclin- 1 endosomes of the nucleus are ubiquitinated (orange); (B,C) 3D reconstruction of the $\mathrm{z}$ stack of Caco2 cells treated with Bin 1 mAb tilted at different angles. The Beclin-1 has a chimney-like (fougaro) structure in the nucleus and the ubiquitinylated peptides are colocalized with Beclin-1 or observed independently after treatment with the Bin1 mAb. Bin1, bridging integrator 1; mAb, monoclonal antibodies

develop fatty liver, without increasing their activity rate or decreasing food intake. Nocturnin affects lipid metabolism or uptake, together with glucose and insulin sensitivity. ${ }^{45,46}$ Sleep deprivation can lead to increased levels of inflammatory cytokines, including IL- $1 \beta$, IL-6, TNF- $\alpha$, and C-reactive protein, which can lead to further activation of the inflammatory cascade..$^{41,47}$

Bin $1 \mathrm{mAb}$ treatment increased the expression of PPARA and PPARG that are involved in regulating inflammation. The genes are also involved in the regulation of TNF- $\alpha$. The expression of peroxisome proliferator-activated receptor gamma, a negative regulator of NF- $\kappa \mathrm{B}$-dependent inflammation, is reduced in the colonocytes of patients with UC, suggesting a causal link to the disease. ${ }^{1}$

The current therapies in UC involve the use of systemic TNF- $\alpha$ inhibitors that lower the circulating levels of TNF$\alpha^{48,49}$ In our studies, we demonstrate that colitis induction upregulates the expression of the genes of many inflammatory cytokines. These cytokines were also increased when probed with our antibody arrays. However, the expression of the genes of the inflammatory cytokines (TNF, IL-1B, IFN- $\gamma$, OSM, and IL-22) was lowered after treatment with the Bin1 mAb. Bin1 mAb activated PPARA, PPARG, and IL-10RA, each known to have anti-inflammatory effects. As an enhanced inflammatory cytokine profile is a hallmark of $\mathrm{UC}$, Bin1 mAb may be used to lower the expression of these cytokines thereby protecting against this disease.
As yet, the mechanism by which antibody enters cells is not known. In this paper, we demonstrated that multiple pathways are involved in the uptake of Bin1 mAb into cells. We used Caco2 cells to determine the mechanism by which the Bin1 mAb is taken into cells. We demonstrated that the Bin $1 \mathrm{mAb}$ is taken up by $\mathrm{Fc}$ gamma RII (CD32). We also observed that endosomes are involved in the uptake of the antibody. The EEA1 endosomes were observed localized to the periphery of groups of cells. The EEA1 endosomes have multiple roles in cells to protect against invading microbes as well as for the transport of molecules into cells. We recently demonstrated that the EEA1 is localized in the mucosal and muscularis layer of the colon. ${ }^{21}$ We now show that the endosomal proteins EEA1, Beclin-1, and sequestosome/p62 are also involved in the uptake of Bin $1 \mathrm{mAb}$.

The cytochromes are involved in the metabolism of drugs. Bin $1 \mathrm{mAb}$ treatment increased the expression of the genes Cyp4f16 (Cytochrome P450), Sult1b involved in drug metabolism. Once the antibody is taken in, it is cleaved into peptides and taken into the nucleus where the fragments are ubiquitinylated and taken out for recycling. Using confocal microscopy we probed for the ubiquitination after treatment with the Bin $1 \mathrm{mAb}$. We took $\mathrm{z}$ stack of $\mathrm{Caco} 2$ and tilted at different angles. We observed ubiquitinated peptides either associated with Beclin-1 or independent of Beclin-1. The Beclin-1 protein was chimney-shaped 
(fougaro; Greek, chimney) with the ubiquitinated peptides associated with it (Figure 8A-C). In our study, the fougaro structures were observed vertically in the nucleus; never horizontal. The fougaro system may be a mechanism to recycle or remove molecules from the nucleus to the external medium. To our knowledge, this is the first report of the fougaro structures composed of Beclin-1 and associated with the ubiquitinated peptides in the nucleus.

Our study both offers a potential new immunotherapeutic for UC and demonstrates that multiple factors may be involved in the development of UC. Lack of sleep or sleep disorders could lead to altered circadian rhythms that could lead to poor lipid metabolism. The altered circadian rhythms could then induce inflammatory cytokines that lead to UC. Earlier, IBD diseases were confined only to older adults and reported to be rare in children. However, currently, the disease is prevalent in children and young adults. ${ }^{50}$ Interestingly, this demographic group is very active in social media, study for longer hours, have a diet rich in sugar and fat and sleep less, factors that may lead to IBD diseases.

\section{ACKNOWLEDGMENTS}

The authors would like to thank the Janssen Research Foundation, the Wawa Foundation and the Women's Board of Lankenau for providing grants for the project. We acknowledge the technical help provided by Mary Carmen Valenzano, Katherine M Diguilio, and James DuHadaway.

\section{CONFLICTS OF INTEREST}

The authors declare no conflicts of interest.

\section{AUTHOR CONTRIBUTIONS}

ST conceived the study and designed the experiments. ST, $\mathrm{KH}$, WA, JG, RD, KW, AK, and JM, performed the experiments. ST, AK, and JM analyzed the data. ST wrote the manuscript. ST, JM, and GCP helped with and edited the manuscript.

\section{ORCID}

\section{Sunil Thomas (1) http://orcid.org/0000-0003-4780-8851}

\section{REFERENCES}

1. Ungaro R, Mehandru S, Allen PB, Peyrin-Biroulet L, Colombel JF. Ulcerative colitis. Lancet. 2017;389:1756-1770.

2. Eaden JA, Abrams KR, Mayberry JF. The risk of colorectal cancer in ulcerative colitis: a meta-analysis. Gut. 2001;48:526-535.
3. Thomas S, Mercado JM, DuHadaway J, DiGuilio K, Mullin JM, Prendergast GC. Novel colitis immunotherapy targets Bin1 and improves colon cell barrier function. Dig Dis Sci. 2016;61:423-432.

4. Tan MS, Yu JT, Tan L. Bridging integrator 1 (BIN1): form, function, and Alzheimer's disease. Trends Mol Med. 2013;19:594-603.

5. Basler M, Dajee M, Moll C, Groettrup M, Kirk CJ. Prevention of experimental colitis by a selective inhibitor of the immunoproteasome. J Immunol. 2010;185:634-641.

6. Langmead B, Salzberg SL. Fast gapped-read alignment with Bowtie 2. Nat Methods. 2012;9:357-359.

7. Ramalingam A, Wang X, Gabello M, et al. Dietary methionine restriction improves colon tight junction barrier function and alters claudin expression pattern. Am J Physiol Cell Physiol. 2010;299:C1028-C1035.

8. Frezza C, Zheng L, Folger O, et al. Haem oxygenase is synthetically lethal with the tumour suppressor fumarate hydratase. Nature. 2011;477:225-228.

9. Love MI, Huber W, Anders S. Moderated estimation of fold change and dispersion for RNA-seq data with DESeq. 2. Genome Biol. 2014;15:550.

10. Wang X, Valenzano MC, Mercado JM, Zurbach EP, Mullin JM. Zinc supplementation modifies tight junctions and alters barrier function of CACO-2 human intestinal epithelial layers. Dig Dis Sci. 2013;58:77-87.

11. Rybakovsky E, Valenzano MC, Deis R, DiGuilio KM, Thomas S, Mullin JM. Improvement of human-oral-epithelial-barrier function and of tight junctions by micronutrients. J Agric Food Chem. 2017;65:10950-10958.

12. Kwon HS, Oh SM, Kim JK. Glabridin, a functional compound of liquorice, attenuates colonic inflammation in mice with dextran sulphate sodium-induced colitis. Clin Exp Immunol. 2008;151:165-173.

13. Chang MY, Boulden J, Valenzano MC, et al. Bin1 attenuation suppresses experimental colitis by enforcing intestinal barrier function. Dig Dis Sci. 2012;57:1813-1821.

14. Ananthakrishnan AN, Khalili H, Konijeti GG, et al. Sleep duration affects risk for ulcerative colitis: a prospective cohort study. Clin Gastroenterol Hepatol. 2014;12:1879-1886.

15. Tsuchida C, Sakuramoto-Tsuchida S, Taked M, et al. Expression of REG family genes in human inflammatory bowel diseases and its regulation. Biochem Biophys Rep. 2017;12:198-205.

16. de Buhr MF, Mähler M, Geffers R, et al. Cd14, Gbp1, and Pla2g2a: three major candidate genes for experimental IBD identified by combining QTL and microarray analyses. Physiol Genomics. 2006;25:426-434.

17. Krämer A, Green J, Pollard J, Jr, Tugendreich S. Causal analysis approaches in ingenuity pathway analysis. Bioinformatics. 2014;30:523-530.

18. Thomas S, Bonchev D. A survey of current software for network analysis in molecular biology. Hum Genomics. 2010;4:353-360.

19. Burczynski ME, Peterson RL, Twine NC, et al. Molecular classification of Crohn's disease and ulcerative colitis patients using transcriptional profiles in peripheral blood mononuclear cells. J Mol Diagn. 2006;8:51-61.

20. Gruenberg J, van der Goot FG. Mechanisms of pathogen entry through the endosomal compartments. Nat Rev Mol Cell Biol. 2006;7:495-504. 
21. Thomas S, Izard J, Walsh E, et al. The host microbiome regulates and maintains human health: a primer and perspective for non-microbiologists. Cancer Res. 2017;77: 1783-1812.

22. Gu J, Congdon EE, Sigurdsson EM. Two novel Tau antibodies targeting the 396/404 region are primarily taken up by neurons and reduce Tau protein pathology. $J$ Biol Chem. 2013;288:33081-33095.

23. Congdon EE, Gu J, Sait HBR, Sigurdsson EM. Antibody uptake into neurons occurs primarily via clathrin-dependent $\mathrm{Fc} \gamma$ receptor endocytosis and is a prerequisite for acute tau protein clearance. J Biol Chem. 2013;288:35452-35465.

24. Walter E, Kissel T. Heterogeneity in the human intestinal cell line Caco-2 leads to differences in transepithelial transport. Eur J Pharma Sci. 1995;3:215-230.

25. DuHadaway JB, Lynch FJ, Brisbay S, et al. Immunohistochemical analysis of Bin1/amphiphysin II in human tissues: diverse sites of nuclear expression and losses in prostate cancer. J Cell Biochem. 2003;88:635-642.

26. Landy J, Ronde E, English N, et al. Tight junctions in inflammatory bowel diseases and inflammatory bowel disease associated colorectal cancer. World J Gastroenterol. 2016;22:3117-3126.

27. Oshima T, Miwa H, Joh T. Changes in the expression of claudins in active ulcerative colitis. $J$ Gastroenterol Hepatol. 2008;23(Suppl 2):S146-S150.

28. Kaplan GG. The global burden of IBD: from 2015 to 2025. Nat Rev Gastroenterol Hepatol. 2015;12:720-727.

29. Ray G. Inflammatory bowel disease in India-past, present, and future. World J Gastroenterol. 2016;22:8123-8136.

30. Ye L, Cao Q, Cheng J. Review of inflammatory bowel disease in China. Scientific World Journal. 2013;2013:296470.

31. Heimerl S, Moehle C, Zahn A, et al. Alterations in intestinal fatty acid metabolism in inflammatory bowel disease. Biochim Biophys Acta. 2006;1762:341-350.

32. Shores DR, Binion DG, Freeman BA, Baker PRS. New insights into the role of fatty acids in the pathogenesis and resolution of inflammatory bowel disease. Inflamm Bowel Dis. 2011;17 (10):2192-2204.

33. Wollny T, Wątek M, Durnaś B, et al. Sphingosine-1-phosphate metabolism and its role in the development of inflammatory bowel disease. Int J Mol Sci. 2017;18(4):741.

34. Murase R, Taketomi Y, Miki Y, et al. Group III phospholipase A2 promotes colitis and colorectal cancer. Sci Rep. 2017;7 (1): 12261.

35. Boulangé CL, Neves AL, Chilloux J, Nicholson JK, Dumas ME. Impact of the gut microbiota on inflammation, obesity, and metabolic disease. Genome Med. 2016;8(1):42.

36. Lumeng CN, Saltiel AR. Inflammatory links between obesity and metabolic disease. J Clin Invest. 2011;121:2111-2117.

37. Lo JC, Ljubicic S, Leibiger B, et al. Adipsin is an adipokine that improves $\beta$ cell function in diabetes. Cell. 2014; 158:41-53.

38. Greenberg AS, Obin MS. Obesity and the role of adipose tissue in inflammation and metabolism. Am J Clin Nutr. 2006;83:461S 465S.
39. Harju AK, Bootorabi F, Kuuslahti M, Supuran CT, Parkkila S. Carbonic anhydrase III: a neglected isozyme is stepping into the limelight. J Enzyme Inhib Med Chem. 2013;28:231-239.

40. Morton NM, Beltram J, Carter RN, et al. Genetic identification of thiosulfate sulfurtransferase as an adipocyte-expressed antidiabetic target in mice selected for leanness. Nat Med. 2016;22:771-779.

41. Kinnucan JA, Rubin DT, Ali T. Sleep and inflammatory bowel disease: exploring the relationship between sleep disturbances and inflammation. Gastroenterol Hepatol. 2013;9:718-727.

42. Swanson GR, Burgess HJ, Keshavarzian A. Sleep disturbances and inflammatory bowel disease: a potential trigger for disease flare? Expert Rev Clin Immunol. 2011;7:29-36.

43. Park YS, Chung SH, Lee SK, et al. Melatonin improves experimental colitis with sleep deprivation. Int $\mathrm{J}$ Mol Med. 2015;35:979-986.

44. Gooley JJ, Chua ECP. Diurnal regulation of lipid metabolism and applications of circadian lipidomics. $J$ Genet Genomics. 2014;41:231-250.

45. Gnocchi D, Pedrelli M, Hurt-Camejo E, Parini P. Lipids around the clock: focus on circadian rhythms and lipid metabolism. Biology. 2015;4:104-132.

46. Green CB, Douris N, Kojima S, et al. Loss of nocturnin, a circadian deadenylase, confers resistance to hepatic steatosis and diet-induced obesity. Proc Natl Acad Sci USA. 2007;104:9888-9893.

47. Testelmans D, Tamisier R, Barone-Rochette G, et al. Profile of circulating cytokines: impact of OSA, obesity and acute cardiovascular events. Cytokine. 2013;62:210-216.

48. Olesen CM, Coskun M, Peyrin-Biroulet L, Nielsen OH. Mechanisms behind efficacy of tumor necrosis factor inhibitors in inflammatory bowel diseases. Pharmacol Ther. 2016;159:110-119.

49. Rutgeerts P, Sandborn WJ, Feagan BG, et al. Infliximab for induction and maintenance therapy for ulcerative colitis. $N$ Engl $J$ Med. 2005;353:2462-2476.

50. Philip M, Augustine P, Thomas V, et al. Kerala inflammatory bowel disease study group. Multi-center prospective survey of inflammatory bowel diseases in Kerala: more than 2000 cases. Indian J Gastroenterol. 2017;36:459-467.

\section{SUPPORTING INFORMATION}

Additional supporting information may be found online in the Supporting Information section at the end of the article.

How to cite this article: Thomas S, Hoxha $\mathrm{K}$, Alexander $\mathrm{W}$, et al. Intestinal barrier tightening by a cell-penetrating antibody to Bin1, a candidate target for immunotherapy of ulcerative colitis.

J Cell Biochem. 2019;120:4225-4237.

https://doi.org/10.1002/jcb.27716 